\title{
Seroprevalence of Hepatitis E Virus among Voluntary Blood Donors in Nairobi County, Kenya: A Pilot Study
}

\author{
Ezekiel Kanue Fardolo1,2, Wallace Bulimo ${ }^{3}$, Patrick Okoti Aluora1, George Gachara ${ }^{1 *}$ \\ ${ }^{1}$ Department of Medical Laboratory Science, School of Medicine, Kenyatta University, Nairobi, Kenya \\ ${ }^{2}$ Department of Medical Laboratory Science, Mother Patern College of Health Sciences, Stella Maris Polytechnic University, \\ Monrovia, Liberia \\ ${ }^{3}$ US-Army Medical Research Directorate, Nairobi, Kenya \\ Email: ^ggachara@gmail.com
}

How to cite this paper: Fardolo, E.K., Bulimo, W., Aluora, P.O. and Gachara, G. (2020) Seroprevalence of Hepatitis E Virus among Voluntary Blood Donors in Nairobi County, Kenya: A Pilot Study. Journal of Biosciences and Medicines, 8, 78-85. https://doi.org/10.4236/jbm.2020.812008

Received: October 27, 2020

Accepted: December 12, 2020

Published: December 15, 2020

Copyright (c) 2020 by author(s) and Scientific Research Publishing Inc. This work is licensed under the Creative Commons Attribution International License (CC BY 4.0).

http://creativecommons.org/licenses/by/4.0/

\begin{abstract}
Hepatitis $\mathrm{E}$ is a common infection caused by the Hepatitis E virus (HEV), a primarily enteric virus. HEV disease is mainly spread via stool contamination of water and food supplies. The virus has recently been identified as a global threat to blood safety. The seroprevalence of HEV among blood donors in Kenya remains unknown. This study was carried out as a pilot study to determine the seroprevalence of the virus among blood donors at Nairobi Blood Transfusion Center during the period from September 2018 to January 2019. A total of three hundred and fifty eight $(\mathrm{n}=358)$ plasma samples were used in this study. The plasma was tested for HEV IgM and IgG antibody using a qualitative membrane-based immunoassay (Biopanda reagents Belfast UK). Demographic characteristics of the blood donors were also collected. The age of the blood donors ranged from 16 to 61 years with a mean age of 29 years $( \pm 14)$. Of the study subjects, $36 \%(n=130)$ were females while $64 \%(n=228)$ were males. Overall, $10.6 \%$ (38) and $7.8 \%$ (28) of the samples were HEV IgG and IgM seropositive respectively. Anti-HEV was distributed among all age groups; however donors aged 20 - 40 years had the highest prevalence. This study shows a relatively high prevalence of anti-HEV among healthy blood donors in Nairobi, Kenya. Further studies would be needed in other population groups to assess the potential benefit of incorporating HEV screening of blood products to the current blood donor selection criteria. Moreover, further studies to determine the circulating genotypes of HEV among this group are required for epidemiological reasons.
\end{abstract}

\section{Keywords}

Hepatitis E Virus, IgG, IgM, Blood Donors, Transmission 


\section{Introduction}

Hepatitis $\mathrm{E}$ is a common acute hepatitis infection caused by the Hepatitis E virus (HEV), a primarily enteric virus. HEV a single-stranded RNA virus has been described as the second leading cause of enteric hepatitis after hepatitis A virus. The virus is a member of the genus Orthohepevirus in the family Hepeviridae [1]. HEV isolates are further grouped into five genotypes all belonging to one serotype. Four of these genotypes are known to infect man. In Africa and Asia, genotypes one and two are highly endemic where they cause waterborne human diseases. These two genotypes exclusively infect humans. Genotypes three and four infect humans, pigs and other animals. They cause sporadic disease in developed countries and have a zoonotic transmission [1] [2]. Genotype 5 infects avian species.

Transmission of HEV primarily occurs by the fecal-oral route via contamination of drinking water and food supplies [3]. Parenteral as well as vertical transmission may also occur particularly in endemic regions; however person to person transmission is uncommon. Zoonotic transmission of $\mathrm{HEV}$ is common in developed countries. Transmission of HEV through blood transfusion and solid organ transplants has also been recently documented (Purpari et al., 2019). Consequently, HEV has been identified as a new global threat to blood safety [4].

HEV causes about twenty million infections yearly worldwide [5], out of which more than three million are asymptomatic. HEV accounts for 55,000 deaths per year, according to the report by the World Health Organization (WHO) in 2015 [6]. A huge proportion of deaths caused by this virus take place in low and medium income countries of Latin America, Asia, and Africa. There have been several outbreaks of HEV in Africa and around the world with varying seroprevalences. A seroprevalence of $42 \%$ was reported in Zambia urban populations [7]. In Egypt, 80\% - 90\% prevalence has also been documented among jaundiced patients [8]. A 14\% seroprevalence was reported among HIV-uninfected people in northern Uganda [9] while in a refugee camp in Eastern Kenya, the prevalence among patients with jaundice was found to be $77.1 \%$ [10].

The viral infection is usually asymptomatic in individuals with an intact immune system. However, it is responsible for disease among immunocompromised patients and pregnant women in sub-Saharan Africa. Based on existing records, approximately $60 \%$ of solid organ transplant recipients exposed to $\mathrm{HEV}$ develop chronic infection, and within 2 years $10 \%$ progress to cirrhosis [11]. The resulting chronic infection may then progress to cirrhosis and death. In Kenya, the country guidelines prescribe the detection of transfusion transmissible pathogens before blood transfusion. The transfusion transmissible infectious pathogens tested among blood donors include syphilis, hepatitis $\mathrm{C}$ virus, hepatitis $B$ virus and human immunodeficiency virus.

$\mathrm{HEV}$ is the most common, yet least diagnosed etiology of acute viral hepatitis. 
However, HEV is currently not tested among potential blood donors in Kenya. The aim of this study was to determine the seroprevalence of hepatitis $E$ virus among voluntary blood donors at the Nairobi blood transfusion centre in Nairobi city county, Kenya.

\section{Methodology}

The sample size was calculated using $N=Z^{2}(p)(1-p) / c^{2} \quad$ (Fisher et al., 1998).

By calculation; $N=1.962(0.77)(1-0.77) \div(0.05)^{2}=3.8416(0.23) \div 0.0025=$ 272.13 (least sample size).

$\mathrm{N}=272.13$.

All 358 archived serum samples used in this study were obtained from the Nairobi regional blood transfusion centre Nairobi, Kenya (Figure 1).

\subsection{Selection and Exclusion Criteria}

The inclusion criteria for the study from whom these samples were collected were voluntary donors who were considered healthy at the time of donation. Potential donors who did not meet the requirements of blood donation as provided by the national blood transfusion services were excluded from the study.

\subsection{Seroepidemiologic Analysis}

Between September 2018 to January 2019, 358 blood samples from volunteer blood donors were collected in a previous study titled "Genotypic characterization of HBV voluntary blood donors in Nairobi regional transfusion center, Kenya" and archived at $-80^{\circ} \mathrm{C}$. The samples were collected at the Nairobi regional blood transfusion center, Nairobi County, Kenya. For the purpose of this study, the plasma from these samples was retrieved and analyzed for HEV.

All the laboratory procedures were carried out at the Kenyatta University main campus laboratories. Plasma samples were tested for both HEV IgM and IgG using an HEV Rapid test cassette (Biopanda reagent Belfast UK). This is a qualitative membrane-based enzyme immunoassay for the detection of HEV antibodies in plasma. The kit has a relative sensitivity and specificity of $90 \%$ and 98.7\% respectively for HEV IgG and 93.3\% and 98.6\% respectively for HEV IgM. In this study, HEV IgM was used as a marker of acute infection. Bivariate statistical analysis was used to determine the association between demographic factors and HEV seropositivity. The variables were considered associated with HEV infection with a $\mathrm{p}$ value of $<0.05$.

\section{Results}

\subsection{HEV Seroprevalence}

Among the 358 archived samples, 130 (36.3\%) were from females while 228 $(63.7 \%)$ were from males (Table 1 ). The ages of the donors ranged from 16 to 61 years with a mean age of 29 years. Out of the three hundred and fifty-eight (358) 


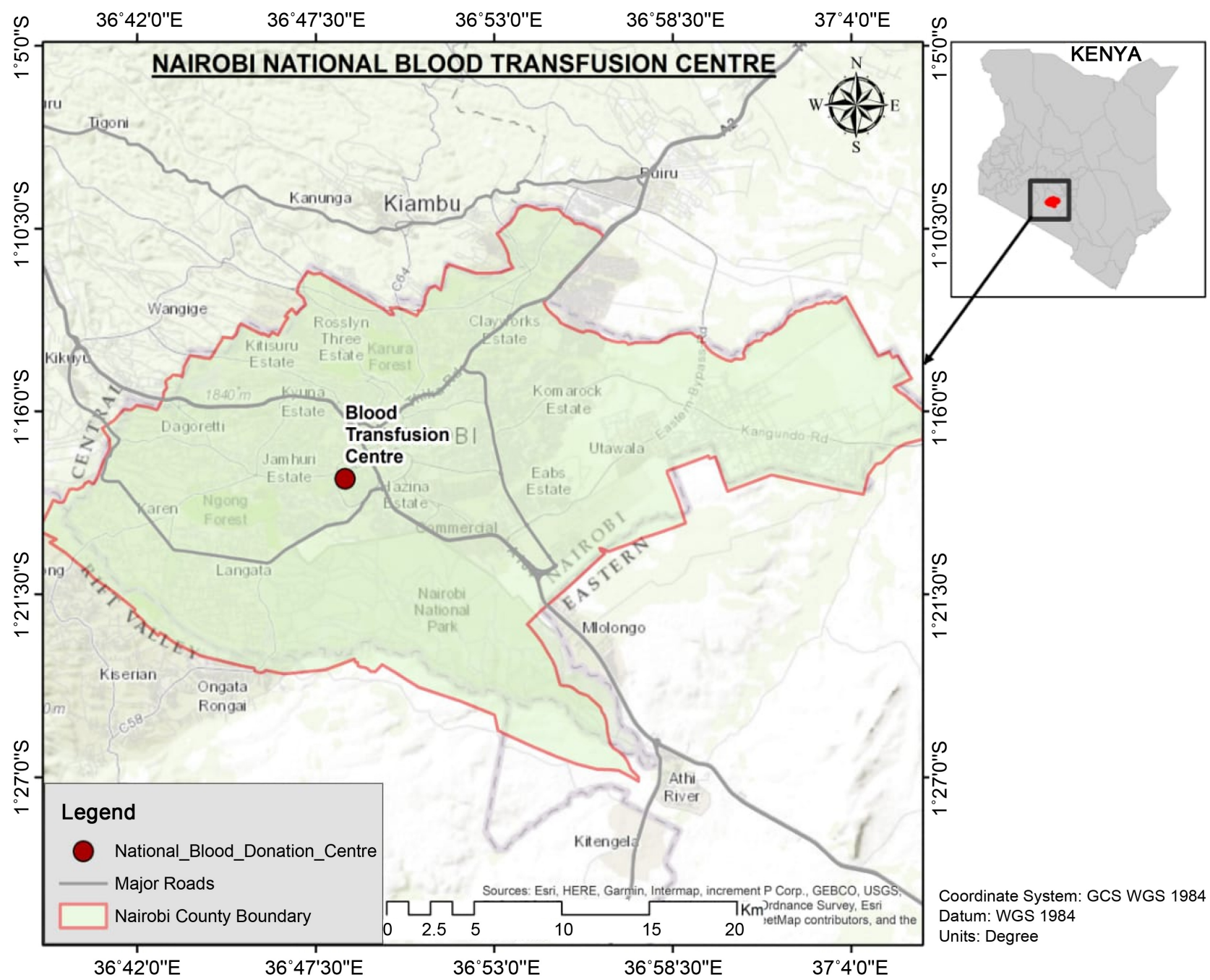

Figure 1. The map of Nairobi county, showing study area.

Table 1. Gender and HEV seropositivity.

\begin{tabular}{cccc}
\hline Sex & Tested samples (\%) & Anti HEV IgM (\%) & Anti HEV IgG (\%) \\
\hline Male & $228(63.7)$ & $19(5.3)$ & $25(7.0)$ \\
Female & $130(36.3)$ & $9(2.5)$ & $13(3.6)$ \\
Total & $358(100)$ & $\mathbf{2 8}(7.8)$ & $38(10.6)$ \\
\hline
\end{tabular}

blood donors examined, 28 (7.8\%) were seropositive for HEV IgM and were thus acutely infected, while 330 (92.2) were negative for HEV IgM. Regarding HEV IgG, 38 (10.6\%) samples were seropositive while 320 (89.4\%) were negative and 27 (7.5\%) samples were seropositive for both IgG and IgM.

\subsection{Association of Demographic Variables with HEV Seropositivity}

The presence of IgM and IgG anti HEV differed by gender $(\mathrm{p}<0.05)$. More males in the study were HEV seropositive than females. The numbers of the 
males with a HEV IgM seropositive reaction were $19(67.9 \%)$ while those with an IgG seropositive reaction were 25 (65.8\%) (Table 2).

The ages of the donors ranged from 16 - 61 years with a mean age of 29 years. The donors were stratified into three age groups: <20 years, 20 to 40 years and $>40$ years. The middle age group had the highest IgM seropositivity; $\mathrm{n}=22$ $(78.6 \%)$, followed by the $>40$ years age group; $n=5(17.9 \%)$. The age groups $<20$ years had the lowest IgM seropositivity. However age was not associated with IgM seropositivity. Similar trends were also shown in regard to IgG seropositivity except that there was observed an association between age and IgG seropositivty.

The highest HEV IgG and IgM seropositivity was observed in donors with a tertiary education and followed by those with a secondary education. Donors with no formal form of educational background showed the lowest seropositivity. There was a significant association between IgM seropositivity and level of education $(\mathrm{P}=0.0338)$.

\section{Discussion}

The detection of HEV antibodies in blood donors' blood is helpful in understanding past and present HEV infection. The current study utilized 358 archived plasma from blood donors and showed that the seroprevalence of HEV among blood donors in Nairobi blood Transfusion centre was $7.8 \%$ and $10.6 \%$ for both anti-HEV IgM and IgG respectively. While HEV prevalence studies among blood donors in Africa are sparse, two studies have reported a seroprevalence range of $4.6 \%$ - 39\% [12]. The seroprevalence in the current study therefore is in agreement with the continental trends. The only other HEV seroprevalence study in Nairobi was conducted in an informal settlement among febrile patients. The study found a higher seroprevalence of $25.7 \%$ (IgM) and

Table 2. Hepatitis E serostatus in relation to demographic characteristics.

\begin{tabular}{|c|c|c|c|c|c|c|}
\hline \multirow{2}{*}{ Characteristic } & IgM Reactive & IgM non-reactive & \multirow{2}{*}{ P-value } & IgG Reactive & IgG non-reactive & \multirow{2}{*}{ P-Value } \\
\hline & $\mathrm{n}=\mathbf{2 8}$ & $\mathrm{n}=330$ & & $\mathrm{n}=\mathbf{3 8}$ & $\mathrm{n}=320$ & \\
\hline Gender & & & 0.0142 & & & 0.0163 \\
\hline Female (\%) & $9(32.1)$ & $121(36.7)$ & & $13(34.2)$ & $117(36.6)$ & \\
\hline Male (\%) & $19(67.9)$ & $209(63.3)$ & & $25(65.8)$ & $203(63.4)$ & \\
\hline Age group (years) & & & 0.757 & & & 0.025 \\
\hline$<20$ & $1(3.6)$ & $47(14.2)$ & & $1(2.6)$ & $46(14.4)$ & \\
\hline $20-40$ & $22(78.6)$ & $249(45.2)$ & & $33(86.8)$ & $239(43.4)$ & \\
\hline$>40$ & $5(17.9)$ & $34(10.3)$ & & $4(10.5)$ & $35(10.9)$ & \\
\hline Level of Education & & & 0.0338 & & & 0.639 \\
\hline Primary & $0(0)$ & $7(2.1)$ & & $0(0)$ & $7(2.2)$ & \\
\hline Secondary & $10(35.7)$ & $77(23.3)$ & & $11(28.9)$ & $76(23.8)$ & \\
\hline Tertiary & $15(53.6)$ & $208(63)$ & & $24(63.2)$ & $199(62.2)$ & \\
\hline None & $3(10.7)$ & $38(11.5)$ & & $3(7.9)$ & $38(11.9)$ & \\
\hline
\end{tabular}


37.8\% (IgG) [3]. It would be expected that healthy blood donors would have a lower seroprevalence compared to febrile patients since $\mathrm{HEV}$ is a known cause of febrile illness. Additionally, the poor sanitation in the informal settlement would lead to a higher prevalence considering HEV is mainly enterically transmitted.

The presence of HEV IgM serves as a marker of recent HEV infection [13]. HEV IgM seropositivity for the current study was $5.3 \%$ in males and 2.5 in females these figures were higher than those obtained from a study in Burkina Faso where IgM seropositivity was $2.6 \%$ in males and $1.9 \%$ in females [14]. However, the low percentages of blood donors with IgM seropositivity indicate that there are low incidences of infection cycles [13].

Demographic data collection was limited to gender, age and the level of education attained by the blood donors. This study determined that both IgG and IgM HEV seroprevalence was higher in males than in females. A meta analysis review of 100 studies in the Middle East and North Africa region found out that HEV seroprevalence in healthy individuals was higher in males than in females [15]. However among diverse study groups, various studies have shown mixed results regarding whether gender predisposes persons to HEV infection [4].

HEV seropositivity has been shown to vary among various age groups. In the current study, the middle age group 20 - 40 years had the highest seroprevalence compared to those aged $<20$ and $>40$ years who had the lowest seroprevalence. This is in contrast to previous studies which document that HEV seroprevalence increases with age but exposure occurs in the younger ages [15]. The higher seroprevalence in the middle age groups in this study could perhaps be explained by the blood donor selection criteria used in the country that relies mostly on school and college going donors who are deemed low risk. Consequently, majority of the blood donors in this study (73\%) were between 20 - 39 years old and this may explain the higher seroprevalence in this age group in the current study. It is expected that if a larger proportion of older blood donors were recruited, perhaps the seroprevalence would increase with age.

Based on results from this study, it was observed that HEV IgM seropositivity was associated with the level of education of the blood donor study population. Majority (24 out of 38) of the IgM seropositive individuals had attained a tertiary level of education. This contradicted a recent study carried out in Ethiopia where the least number of seropositivity was among those who had attained a tertiary level of education (22\%) and the highest was among those who had attained a primary school level of education (36\%) [8]. Perhaps this is an indictment of the sanitation standards in majority of the middle class neighborhoods in Nairobi where those with a tertiary education mostly reside.

\section{Conclusion}

The epidemiology of HEV among blood donors is important in evaluating blood safety. The $7.8 \%$ HEV IgM seroprevalence in this study is high considering that 
the potential blood donors selected are considered low risk. Further studies should be conducted in this group to determine the other predisposing risk factors for HEV infection as well as the circulating genotypes of HEV in Kenya.

\section{Acknowledgements}

I would like to first express my appreciation to the Mother College of Health Sciences, Stella Maris Polytechnic University for her support. Secondly, my sincere thanks and appreciation go to Ruth Maundu and Abdul A. Jalloh who have been my great source of inspiration.

\section{Conflicts of Interest}

The authors declare no conflicts of interest regarding the publication of this paper.

\section{References}

[1] Zhang, X., Bilic, I., Troxler, S. and Hess, M. (2017) Evidence of Genotypes 1 and 3 of Avian Hepatitis E Virus in Wild Birds. Virus Research, 228, 75-78. https://doi.org/10.1016/j.virusres.2016.11.028

[2] Woo, P.C., Lau, S.K., Teng, J.L., Cao, K.Y., Wernery, U., Schountz, T. and Yuen, K.Y. (2016) New Hepatitis E Virus Genotype in Bactrian Camels, Xinjiang, China, 2013. Emerging Infectious Diseases, 22, 2219-2221. https://doi.org/10.3201/eid2212.160979

[3] Furukawa, N.W., Teshale, E.H., Cosmas, L., Ochieng, M., Gikunju, S., Fields, B.S. and Montgomery, J.M. (2016) Serologic Evidence for Hepatitis E Virus Infection among Patients with Undifferentiated Acute Febrile Illness in Kibera, Kenya. Journal of Clinical Virology, 77, 106-108. https://doi.org/10.1016/j.jcv.2016.02.021

[4] Kim, J.-H., Nelson, K.E., Panzner, U., Kasture, Y., Labrique, A.B. and Wierzba, T.F. (2014) A Systematic Review of the Epidemiology of Hepatitis E Virus in Africa. BMC Infectious Diseases, 14, 308. https://doi.org/10.1186/1471-2334-14-308

[5] Kmush, B.L., Labrique, A.B., Dalton, H.R., Ahmed, Z.B., Ticehurst, J.R., Heaney, C.D. and Zaman, K. (2015) Two Generations of "Gold Standards": The Impact of a Decade in Hepatitis E Virus Testing Innovation on Population Seroprevalence. The American Journal of Tropical Medicine And Hygiene, 93, 714-717. https://doi.org/10.4269/ajtmh.15-0159

[6] Guo, Y., Feng, Y., Qu, F., Zhang, L., Yan, B. and Lv, J. (2020) Prediction of Hepatitis E Using Machine Learning Models. PLoS ONE, 15, e0237750. https://doi.org/10.1371/journal.pone.0237750

[7] Jacobs, C., Chiluba, C., Phiri, C., Lisulo, M.M., Chomba, M., Hill, P.C. and Kelly, P. (2014) Seroepidemiology of Hepatitis E Virus Infection in an Urban Population in Zambia: Strong Association with HIV and Environmental Enteropathy. Journal of Infectious Diseases, 209, 652-657. https://doi.org/10.1093/infdis/jit409

[8] Abebe, M., Ali, I., Ayele, S., Overbo, J., Aseffa, A. and Mihret, A. (2017) Seroprevalence and Risk Factors of Hepatitis E Virus Infection among Pregnant Women in Addis Ababa, Ethiopia. PLoS ONE, 12, e0180078.

https://doi.org/10.1371/journal.pone.0180078

[9] Boon, D., Redd, A.D., Laeyendecker, O., Engle, R.E., Nguyen, H., Ocama, P. and Gray, R.H. (2018) Hepatitis E Virus Seroprevalence and Correlates of Anti-HEV 
IgG Antibodies in the Rakai District, Uganda. The Journal of Infectious Diseases, 217, 785-789. https://doi.org/10.1093/infdis/jix610

[10] Ahmed, J.A., Moturi, E., Spiegel, P., Schilperoord, M., Burton, W., Kassim, N.H. and Burke, H. (2013) Hepatitis E Outbreak, Dadaab Refugee Camp, Kenya, 2012. Emerging Infectious Diseases, 19, 1010-1011.

https://doi.org/10.3201/eid1906.130275

[11] Lhomme, S., Abravanel, F., Dubois, M., Sandres-Saune, K., Rostaing, L., Kamar, N. and Izopet, J. (2012) Hepatitis E Virus Quasispecies and the Outcome of Acute Hepatitis E in Solid-Organ Transplant Patients. Journal of Virology, 86, 10006-10014. https://doi.org/10.1128/JVI.01003-12

[12] Maponga, T.G., Lopes, T., Cable, R., Pistorius, C., Preiser, W. and Andersson, M.I. (2020) Prevalence and Risks of Hepatitis E Virus Infection in Blood Donors from the Western Cape, South Africa. VoxSanguinis. https://doi.org/10.1111/vox.12966

[13] Stramer, S.L., Moritz, E.D., Foster, G.A., Ong, E., Linnen, J.M., Hogema, B.M. and Dodd, R.Y. (2016) Hepatitis E Virus: Seroprevalence and Frequency of Viral RNA Detection among US Blood Donors. Transfusion, 56, 481-488.

https://doi.org/10.1111/trf.13355

[14] Traore, K.A., Ouoba, J.B., Rouamba, H., Nebie, Y.K., Dahourou, H., Rossetto, F. and Roques, P. (2016) Hepatitis E Virus Prevalence among Blood Donors, Ouagadougou, Burkina Faso. Emerging Infectious Diseases, 22, 755-757. https://doi.org/10.3201/eid2204.151728

[15] Al-Sadeq, D.W., Majdalawieh, A.F. and Nasrallah, G.K. (2017) Seroprevalence and Incidence of Hepatitis E Virus among Blood Donors: A Review. Reviews in Medical Virology, 27, e1937. https://doi.org/10.1002/rmv.1937 